\title{
Ciudad, ecología, clima y bien común: estado de situación, retos y perspectivas desde América Latina ${ }^{1}$
}

\section{City, ecology, climate and common good: status of the situation, challenges and perspectives from Latin America}

\author{
Gian Carlo Delgado Ramos \\ Doctorado en Ciencias Ambientales. \\ Universidad Nacional Autónoma de México \\ giandelgado@unam.mx
}

\section{Resumen}

El trabajo abre con una revisión de la problemática urbana en general y en América Latina, para luego dar cuenta de las principales implicaciones y retos inmediatos y a mediano plazo, así como las potenciales soluciones actualmente en discusión, sobre todo desde la perspectiva del metabolismo urbano y la ecología política urbana, o mejor dicho, de la ecología política del metabolismo urbano. Se analizan así los perfiles metabólicos de algunas ciudades latinoamericanas, incluyendo algunas cuestiones relacionadas a las implicaciones climáticas y socioambientales. Asimismo se revisa hasta dónde las principales soluciones propuestas en la literatura internacional y en la academia, contribuyen con la construcción de alternativas anti-sistémicas o, en su defecto, hasta dónde resultan ser apuestas de resistencia a cualquier cambio profundo de paradigma, que en el caso de lo urbano alude desde la perspectiva aquí asumida, a ciudades cuya función -espacial/territorial- se aleja de la acumulación de capital para en cambio construir, con acciones coordinadas y consensuadas de abajo hacia arriba y de arriba hacia abajo, asentamientos más justos, sustentables y resilientes, con relaciones más armónicas con los territorios aledaños y más allá.

Palabras clave: metabolismo urbano, ecología política urbana, cambio climático, sustentabilidad urbana, política pública.

\begin{abstract}
The work opens with a review of the overall urban problematic and in Latin America, to further give account of the main implications and immediate and midterm challenges, as well as the potential solutions currently under discussion, especially from the urban metabolism perspective and the urban ecology policy, better said, of the political ecology of urban metabolism. Thus the metabolic profiles of some Latin American cities are analyzed, including some matters related to the climate and socioenvironmental implications. Likewise, how far the main solutions proposed in international literature and in the academy contribute to the construction of antisystemic alternatives, or by default, how far are they bets of resistance to any deep paradigm change, is reviewed, in case of the urban it refers from the approach assumed here, to cities which spatial/territorial function- moves away from the accumulation of capital to rather build, with coordinated and consensus actions from bottom to top and from top to bottom, more fair, sustainable and resilient settlements, with more harmonic relations with the adjacent territories and beyond.
\end{abstract}

Key Words: urban metabolism, urban ecology policy, climate change, urban sustainability, public policy.

\section{Introducción}

Hoy día 52\% de la población mundial es urbana. Proyecciones para el 2050 indican que ésa podría ubicarse entre el 64\% y 69\% de la población total mundial (Naciones Unidas, 2011), momento en el que la extensión de la capa urbana se duplicaría o hasta triplicaría, dependiendo de las dinámicas poblacionales y económicas (Angel et al, 2011; IPCC, 2014). En tal escenario, la urbanización será más intensa en regiones que aún no han experimentado tasas importantes de urbanización y que al mismo tiempo prometen al menos un relativo crecimiento económico futuro (Naciones Unidas, 2011). Esto es, en Asía países como China e India esencialmente, pero también ciertas regiones de África que se colocan en esta dinámica pues al día de hoy tales continentes registran, en promedio, sólo un 45\% y 40\% de población total urbanizada respectivamente (Ibid).

1 - Texto derivado de los resultados del proyecto investigación CEIICH-PINCC sobre "Valoración del metabolismo urbano en la Ciudad de México y sus impactos socioeconómicos frente al cambio climático" terminado en Julio de 2014. 
No es menor precisar que tal proceso de urbanización ha sido histórica y geográficamente desigual pues el grueso de asentamientos irregulares, que suman el $32 \%$ de la población mundial urbana, están en los países pobres (Davis, 2006). En promedio el 43\% de la población urbana de los países en desarrollo vive en dichos espacios, pero casos como el Chad, Etiopia o Afganistán, rondan el 98\% - 99.4\%; en contraste, en los países ricos, los barrios pobres cubren en promedio tan sólo el $6 \%$ de sus espacios urbanos (Ibid). ${ }^{2}$

En este panorama, es notorio que América Latina (AL) sea la única región del mundo "en desarrollo" con índices que promedian $78 \%$ de población urbana (UNHABITAT, 2009). El porcentaje de población que viven en zonas de alta marginación es del $27 \%$ en promedio (Ibid), aunque es variable pues se estima en el orden del $19.6 \%$ en México; en $36.6 \%$ en Brasil; $33.1 \%$ en Argentina; $68 \%$ en Perú, por dar algunos ejemplos (Davis, 2006).

Dado el tipo de economía que caracteriza a la región, dígase primario-exportadora y en el mejor de los casos maquiladora, pero también a la inestabilidad de la misma y a los altos índices de inequidad (Delgado, 2011), el resultado de tal dinamismo urbano es que las ciudades latinoamericanas figuran como íconos representativos de inmensos y típicos (des)ordenamientos territoriales y con patrones de expansión han sido acelerados $y$, aunque los ritmos de urbanización de la región han decaído en los últimos años (la tasa de crecimiento anual promedio ronda el $1.8 \%$ ), se espera sin embargo que ésa siga creciendo hasta alcanzar el $87 \%$ de población urbana para 2050. Además son ciudades excluyentes que representan un espacio de oportunidades sólo para algunos de sus habitantes.

Tomando nota de este escenario, a continuación se presenta una aproximación a los perfiles de consumo de materiales y energía de los asentamientos urbanos, metodología también conocida como metabolismo urbano; el caso se aterriza para algunas ciudades de América Latina. Posteriormente se analizan los conflictos de acceso, gestión y usufructo de los recursos, incluyendo la tierra, las asimetrías presentes y los intereses creados que están detrás de la conformación de las funciones del propio territorio urbano, su lógica y finalidad, dígase en beneficio de qué y de quién. El caso de la generación y gestión de los residuos en la Ciudad de México es expuesto para propósitos explicativos.

Finalmente se aborda la necesidad del planteo de nuevos paradigmas urbanos, no sólo de aquellos típicamente reconocidos en el ámbito de especialistas diversos, sino también de otros que abogan por un genuino buen vivir urbano y en general por el bien común de la humanidad (Daiber y Houtart, eds., 2012; Delgado, coord., 2014, entre otros). En este último punto se sostiene que el metabolismo urbano, como potente herramienta analítica del ámbito biofísico, acompañado en todo momento de una visión crítica de lo socioeconómico, ambiental y político, como la que caracteriza a la ecología política (urbana), puede contribuir al proceso de construcción de alternativas y de rutas de transición. Se trata de un punto que, en efecto, obliga la discusión sobre el significado de lo alternativo, esto es, preguntarse, alternativo a qué.

\section{Metabolismo urbano, sustentabilidad y clima: una mirada de sus múltiples enfoques desde América Latina.}

Las ciudades toman cuerpo en territorios concretos, con características biofísicas naturales específicas -o primera naturaleza-, sobre los cuales se conforma una segunda naturaleza (Mumford, 1961, Lefebvre, 1976) que materialmente demanda intercambios de materia (biótica, abiótica, de origen natural o antrópico) y energía (renovable o no-renovable) con sus alrededores o hinterlandy más allá. Tales intercambios se dan en dos sentidos, en términos de flujos de materiales y energía que entran a los asentamientos urbanos y en flujos de materiales y de energía degradados que salen. Y dado que los flujos de salida son diversos no sólo en términos de composición biofísica sino en cuanto a su tiempo de vida útil, se habla así de la conformación de un stock de materiales (y de energía incorporada, o la cantidad de energía usada para su producción e incorporación en lo que en sí conforma la infraestructura urbana y el parque vehicular). Véase Figura 1. Lo anterior se observa desde tres componentes generales: (1) flujos y conformación de stocks de materia y energía, (2) procesos mediante los cuales ésos toman cuerpo, y (3) la sociedad en tanto que, mediante relaciones sociales de producción específicas, define tales o cuales perfiles metabólicos y construye el espacio territorial concreto.

El estudio interdisciplinar de los tres componentes descritos es una cuestión cada vez más relevante, no sólo debido al creciente uso de recursos por parte de los asentamientos urbanos, sino también en tanto que permite hacer lecturas más finas del estado de situación actual de tales o cuales asentamientos en términos biofísicos, así como de su proyección futura, lo que a su vez abre la posibilidad de modelar rutas más, o menos, eficientes en el uso de los recursos y en la generación de residuos y así enfocar esfuerzos. Dígase por ejemplo, por medio de la planificación de los procesos metabólicos, desde la definición de las funciones y uso del territorio así como del mismo diseño de tales o cuales infraestructuras con base en la relevancia otorgada para su optimización, dígase en

\footnotetext{
2 - El proceso de ampliación de las zonas urbanas de alta marginación se acentuó a partir de la década de 1990 cuando se registró un aumento de 36\% de la población ahí emplazada, misma que, de seguir la actual tendencia, se duplicará para el 2040 (Davis, 2006).

3 - En relación al metabolismo entre la sociedad y la naturaleza, Toledo, Alarcón-Cháires y Barón (2009: 334 ) sostienen que, ése: “...implica el conjunto de procesos por medio de los cuales los seres humanos organizados en sociedad, independientemente de su situación en el espacio (formación social) y en el tiempo (momento histórico) se apropian, circulan, transforman, consumen y excretan materia y/ energía provenientes del mundo natural".
} 
términos socioambientales, climáticos y/o de salud pública. Se trata de una línea de análisis que, pese a su importancia, sigue siendo muy limitada en la literatura.

Figura 1. Esquematización del metabolismo urbano.

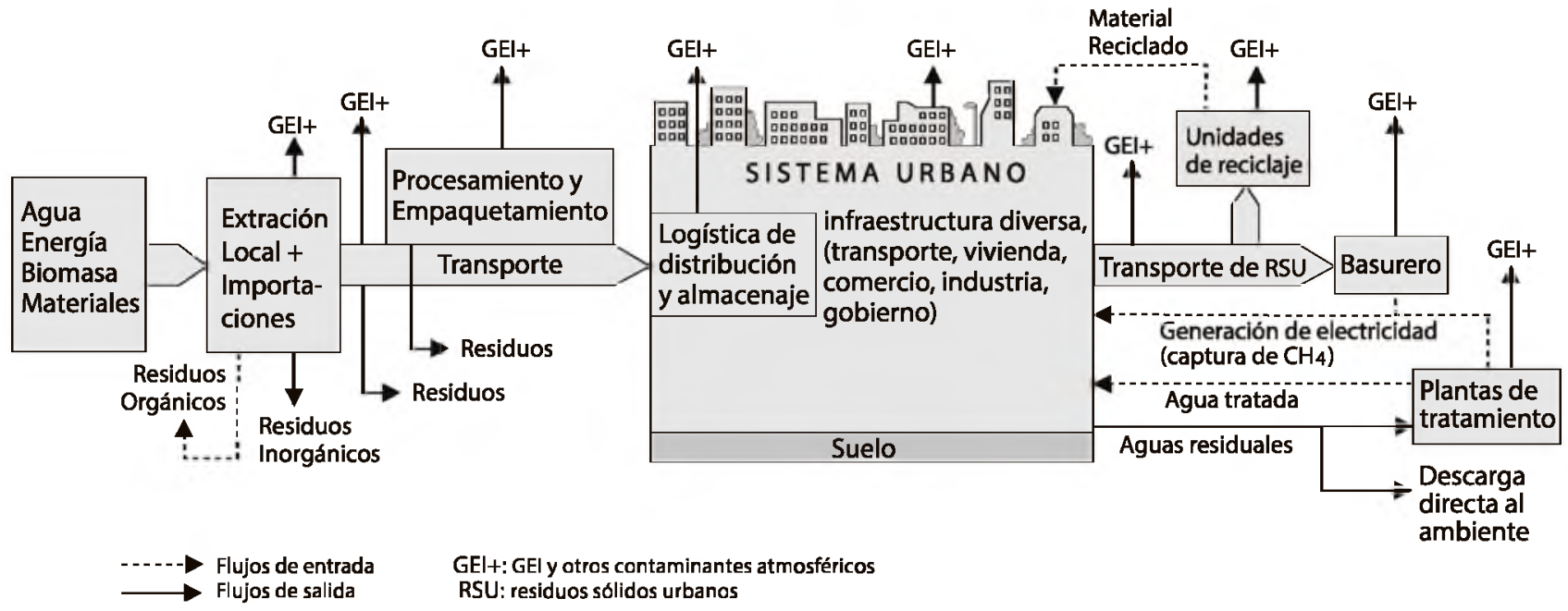

Fuente: elaboración propia. Diseño gráfico: Ángeles Alegre Schettino.

El metabolismo urbano tiene sus orígenes empíricos en el trabajo de Wolman $(1965)^{4}$ y más adelante en las corrientes propias de la economía ecológica y sobre todo en la ecología industrial (Ayres, 1994) que se avocaron a la cuestión urbana a partir de aplicar métodos de análisis de flujos de materiales o MFA por sus siglas en Inglés (para una revisión técnica, léase: Brunner y Rechberger, 2005) y poco después flujos de energía o lo que Haberl (1997 y 2001) calificó como "metabolismo energético".

El enfoque inicial fue el estudio exclusivo de flujos de materiales, seguido de los de energía (modelo metabólico linear), para después incorporar una visión o modelo cíclico de los flujos, es decir, aquella que incorpora la noción de "ciclos cerrados de materiales" o de procesos de reciclaje y/o recuperación parcial de flujos de salida, por ejemplo de materiales valiosos en los residuos sólidos, de gas metano emitido por rellenos sanitarios, de agua tratable, etcétera). Más adelante se incorporaría por parte de diversos autores el análisis de los componentes internos de cada flujo como redes de procesos (Zhang, 2013). En todos los casos existen dos modalidades de construcción de datos, de arriba hacia abajo (generalizaciones de promedios nacionales o regionales a nivel local) o de abajo hacia arriba (minado de dato en los territorios concretos), siendo este último el más preciso pero el más difícil de construir de manera dinámica o multitemporal en tanto que a nivel urbano suele haber muchas deficiencias de datos, ya no se diga problemas de su calidad.

El grueso de análisis metabólicos han sido realizados para ciudades de países desarrollados y enfocándose sobre todo, en varios o ciertos flujos metabólicos (agua, alimentos, energía, etcétera). La contribución de Baccini y Bruner (1990 y 2012) como precursores teórico-metodológicos contemporáneos y, más delante de otros como Kennedy et al (2007, 2009 y 2011), Minx et al (2010), entre otros, son destacables pues permiten tener una visión amplia e integrada de la evolución de los estudios sobre metabolismo urbano, incluyendo las contribuciones climáticas de éstos. En especial el trabajo de Kennedy et al (2011)y Zhang (2013) son referencias obligadas en tanto que ofrece un meta-análisis de las publicaciones en el área y sus metodologías y enfoques.

4 - El autor analiza los flujos de entrada y salida de energía y materiales de una ciudad hipotética de EUA de un millón de habitantes, advirtiendo correctamente la complejidad y variabilidad de ésos. Identificó tres flujos clave de entrada (agua, alimentos y combustible) y tres de salida (aguas residuales, residuos sólidos y contaminantes atmosféricos). Dicha ciudad hipotética consumía 625 mil toneladas diarias de agua y generaba 500 mil toneladas diarias de aguas residuales; asimismo, el consumo de combustible era de unas 9,500 toneladas diarias y el de alimentos de unas ? mil toneladas al día (Wolman, 1965). La conclusión a la que llega el estudio es que llegada una cierta intensidad metabólica, se tornan inevitables los problemas ambientales, la contaminación atmosférica y la incapacidad de gestionar enteramente los residuos urbanos, sobre todo las aguas residuales (Ibid).

5 - Los estudios MEFA parten con delimitar los límites de análisis, para después identificar los flujos existentes y clasificarlos, y así concluir con una matriz de balance que los contabiliza. Si existe suficiente información de calidad en el tiempo, es posible monitorear los flujos y stocks de materiales a lo largo de todo su ciclo de vida.

6 - Entre la diversidad de estudios realizados cabe mencionar, por ejemplo, para flujos energéticos de ciudades como Tokio (Hanya y Ambe, 1976), Hong Kong (Newcombe et al, 1978), Miami (Zucchetto, 1975), Taipei (Huang, 1998), Paris (Barles, 2007 y 2009), Bogotá (Díaz-Álvarez, 2011) y ciertas capitales de América Latina (Delgado et al, 2012, Delgado, 2013). Otras entregas se han en cambio enfocado en el metabolismo urbano del agua (Hermanowicz y Asano, 1999; Gandy, 2004; Thériault et al, 2001; Delgado, 2014); el del ciclo de nutrientes del suelo (Forkes, 2007 ;; Færge et al, 2001); o en el de residuos (Leach, Bauen y Lucas, 1997; Lehmann, 2011). Entre muchos más. 
Resultados de un modelo linear del metabolismo urbano de algunas de las principales ciudades capitales de AL devela que si se considera la población como único criterio, las zonas metropolitanas de Buenos Aires y Rio de Janeiro deberían ser metabólicamente hablando similares, mientras que lo mismo aplicaría para la ZMVM y Sao Paulo. Desde luego, las condiciones biofísicas, el tipo de uso de suelo y otras características -incluyendo aquellas de tipo cultural-, producen variaciones importantes (véase Cuadro 1), no obstante lo que se corrobora es un aumento del metabolismo urbano. El caso de la Ciudad de México en materia de energía es revelador en tanto que pasó de un consumo de unos $443 \mathrm{Pj}$ de energía en 1990 a $545 \mathrm{Pj}$ en 2006 y, en 2010, a $527 \mathrm{Pj}$ de consumo energético fósil y $179 \mathrm{Pj}$ de energía eléctrica producida fuera de la ciudad (que no era contemplada en la medición de 2006) (SMA-DF, 2012). En Montevideo, mientras la población no aumentó de 1996 al 2013, el consumo de energía lo hizo en $40 \%$ (con base en datos de consulta directa a la Intendencia de Montevideo, 1 de Octubre de 2014). ${ }^{7}$

Cuadro 1. Patrones de flujos metabólicos de algunas ciudades latinoamericanas.

\begin{tabular}{|c|c|c|c|c|c|c|c|c|c|c|}
\hline \multirow[b]{2}{*}{ Ciudad } & \multirow{2}{*}{ 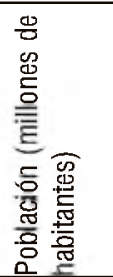 } & \multirow{2}{*}{ 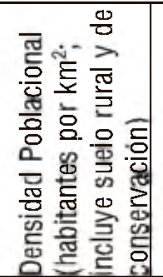 } & \multirow[b]{2}{*}{ 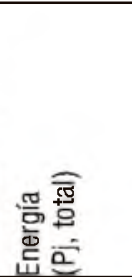 } & \multicolumn{2}{|c|}{$\begin{array}{l}\text { Agua Potable (litros } \\
\text { per cápita/día) }\end{array}$} & \multicolumn{2}{|c|}{$\begin{array}{l}\text { Alimentos } \\
\text { (per cápita/día) }\end{array}$} & \multirow{2}{*}{ 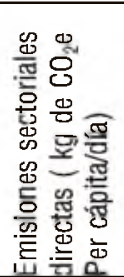 } & \multirow{2}{*}{ 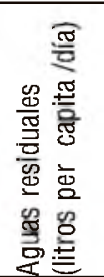 } & \multirow{2}{*}{ 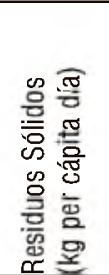 } \\
\hline & & & & 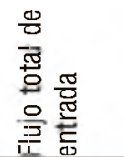 & 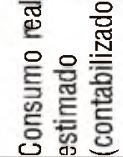 & 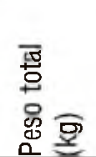 & 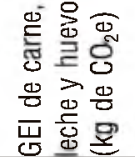 & & & \\
\hline $\begin{array}{l}\text { Ciudad de } \\
\text { México } \\
\text { (ZMVM) }\end{array}$ & $\begin{array}{l}8.85 \\
\left(22^{\star}\right)\end{array}$ & $\begin{array}{l}6,020 \\
\left(2,845^{\star}\right)\end{array}$ & $706^{*}$ & 327 & 220 & $2.01^{\star}$ & $1.98^{*}$ & $6.8^{\star}$ & $\sim 390$ & 1.4 \\
\hline $\begin{array}{l}\text { Sao } \\
\text { Paulo } \\
\text { (Metro) }\end{array}$ & $\begin{array}{l}11.31 \\
\left(20^{\star}\right)\end{array}$ & $\begin{array}{l}7,492 \\
\left(2,492^{\star}\right)\end{array}$ & $\sim 277.8$ A & 290 & $\begin{array}{l}186.8 \\
191\end{array}$ & 1.99 & 2.91 & 4.1 & 120 & 0.93 \\
\hline $\begin{array}{l}\text { Lima } \\
(+ \text { Callao })\end{array}$ & $\begin{array}{l}8.6 \\
\left(9.6^{\star}\right)\end{array}$ & $\begin{array}{l}3,225 \\
\left(3,405^{\star}\right)\end{array}$ & $\begin{array}{l}185- \\
200 * 1 \\
\end{array}$ & $\begin{array}{l}234- \\
250^{*}\end{array}$ & $150^{*}$ & 1.72 & 0.70 & 14.1 & 196 & 1.86 \\
\hline $\begin{array}{l}\text { Bogotá } \\
\text { D. C. }\end{array}$ & $\begin{array}{l}7.3 \\
\left(9.85^{\star}\right)\end{array}$ & $\begin{array}{l}4,599 \\
\left(5,687^{\star}\right)\end{array}$ & 228.7 & 428 & 171 & 1.82 & 1.99 & 5.17 & 192 & 0.76 \\
\hline $\begin{array}{l}\text { Rio de } \\
\text { Janeiro }\end{array}$ & $\begin{array}{l}6.35 \\
\left(11^{\star}\right)\end{array}$ & $\begin{array}{l}5,250 \\
\left(1,948^{\star}\right)\end{array}$ & $\begin{array}{l}\sim 161 \\
\wedge\end{array}$ & 472 & $\begin{array}{l}237.8 \\
226\end{array}$ & 1.99 & 2.91 & 1.9 & 170 & 0.98 \\
\hline $\begin{array}{l}\text { Buenos } \\
\text { Aires } \\
\text { (Gran BAs) }\end{array}$ & $\begin{array}{l}3 \\
\left(12^{\star}\right)\end{array}$ & $\begin{array}{l}14,778 \\
\left(3,130^{\star}\right)\end{array}$ & 337.8 & $535^{\star}$ & $370^{\star}$ & 2.06 & 4.31 & 9.04 & $500^{*}$ & 1.66 \\
\hline $\begin{array}{l}\text { Caracas } \\
\text { Capital } \\
\text { (AMC) }\end{array}$ & $\begin{array}{l}2.1 \\
\left(3^{\star}\right)\end{array}$ & $\begin{array}{l}4,910 \\
\left(3,771^{\star}\right)\end{array}$ & --- & $\begin{array}{l}\sim 509 \\
\left(\sim 620^{\star}\right)\end{array}$ & $\begin{array}{l}400 \\
\left(474^{\star}\right)\end{array}$ & 1.97 & 2.38 & 3.5 & --- & $\begin{array}{l}0.99- \\
1.2\end{array}$ \\
\hline Quito & $\begin{array}{l}1.6 \\
\left(2.2^{*}\right)\end{array}$ & $\begin{array}{l}4,545 \\
\left(527^{\star}\right)\end{array}$ & $\sim 50.1^{*}$ & $271^{*}$ & $189^{*}$ & 1.41 & 1.76 & $\begin{array}{l}-13 \\
\left(25.7^{\star}\right)\end{array}$ & --- & 0.73 \\
\hline Monte-video & $\begin{array}{l}1.3 \\
\left(1.7^{\star}\right)\end{array}$ & 2,488 & $\sim 97 \%$ & 368 & 181 & 2.14 & 2.99 & $8.1-8.2$ & $\cdots$ & 1.4 \\
\hline \multicolumn{11}{|c|}{ 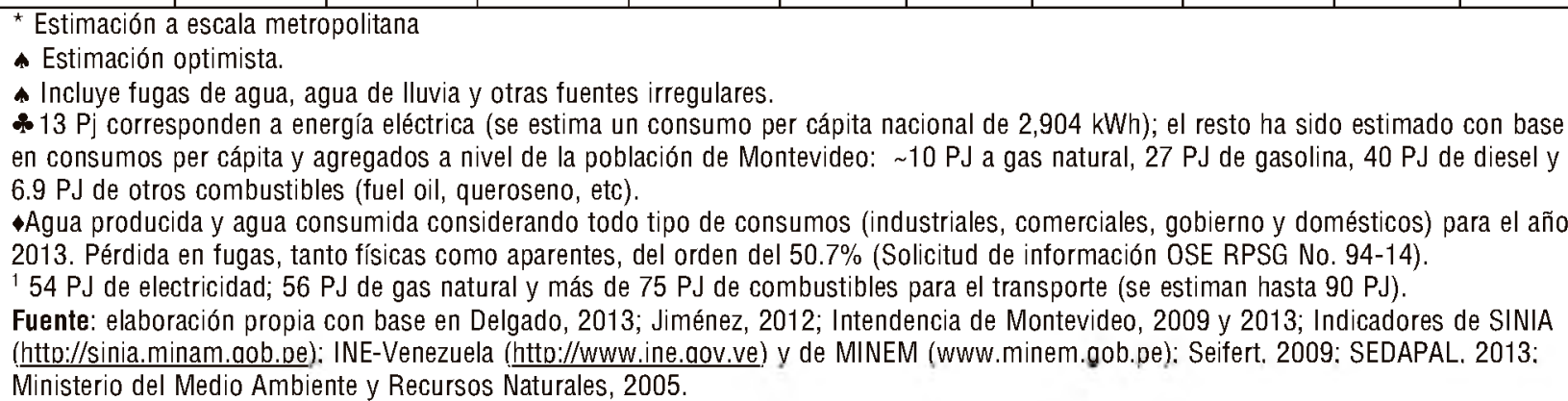 } \\
\hline
\end{tabular}

7 - El consumo total de energía eléctrica en 1996 era de 2.59 millones de MWh, para el 2013 dicho consumo alcanzó los 3.65 millones de MWh. En ese mismo periodo, la población pasó de 1,344,839 habitantes en 1996 a unos 1,319,108 habitantes en 2011 (se estima una población similar para el 2013; con base en datos de www.ine.gub.uy). 
Derivado del análisis preliminar sintetizado en el Cuadro 1, puede afirmarse que las ciudades capitales de AL estudiadas enfrentan grandes retos asociados a la obtención de energía, sobre todo de aquella destinada al transporte $y$, en un segundo plano, también para la generación de electricidad.

En el sector transporte los avances de mayor impacto tanto en el consumo energético como en la reducción de emisiones asociadas, tendrían que gestarse en el marco de un cambio de paradigma de la movilidad urbana, hacia uno más integrado, eficiente, de tipo masivo, pero también no motorizado y vinculado al uso mixto del suelo y a procesos de re-densificación (Delgado, coord.., 2012).

En lo que respecta a los flujos de entrada de agua, se observa una disponibilidad del líquido relativamente segura para las ciudades estudiadas con excepción de la Zona Metropolitana del Valle de México (ZMVM) que ya se encuentra en un estado de alto estrés hídrico; sin embargo, la calidad del líquido sí es en diversos grados una variable que requerirá aún más atención tanto para la ZMVM, como el Gran Buenos Aires (Delgado et al, 2012). Todo esto en efecto conlleva a un uso energético importante por lo que las medidas deberán ser bien diseñadas, ahorrativas y eficientes, e idealmente haciendo uso de energía renovable. Por otro lado, si bien las aguas residuales son en su mayor parte tratadas en las dos megaciudades brasileñas estudiadas (al menos en un cierto grado), este no es el caso de Buenos Aires y la Ciudad de México, por lo que ahí se requiere implementar acciones crecientes en dicho rubro (Ibid).

En el caso de la generación de residuos uno de los principales retos es la disminución de los patrones de consumo, no sólo porque se corrobora que mientras más grande es la población mayor es el valor de generación per cápita de residuos (INECC, 2012), sino también porque en términos climáticos la disminución del consumo de productos y por tanto de generación de residuos se convierten en la principal medida de mitigación esto es, en términos de residuos y emisiones evitadas. La Ciudad de México y Buenos Aires enfrentan tal vez el mayor reto al colocarse a la cabeza en la generación de residuos en términos per cápita. Por su parte, en la Ciudad de México y Sao Paulo, debido al gran volumen generado, la gestión de residuos debería aumentar el reciclaje e impulsar la composta y generación de biogás para la producción de energía eléctrica. Esto último una acción ciertamente desaprovechada para el caso de la Ciudad de México pero no así en el caso de Sao Paulo donde es una de las principales medidas de mitigación del plan de acción climática de la ciudad.
Ahora bien, no es sino hasta tiempos recientes que el análisis del stock urbano comenzó a incluirse como aspecto clave de los estudios metabólicos urbanos, ello en tanto que la energía incorporada de los materiales que lo componen es muy elevada, dígase especialmente acero, cemento y aluminio. ${ }^{8}$ En ese sentido, el trabajo de Müller et al (2013) es valioso pues estima las dimensiones del stock que compone la infraestructura global en términos de $\mathrm{CO}_{2}$ e derivado de la energía incorporada en los tres materiales antes mencionados. Los autores concluyen que la infraestructura global representa $122(-20 /+15)$ gigatoneladas de $\mathrm{CO}_{2}$ e para el caso de los 3 materiales en cuestión, de las cuales 68 gigatoneladas corresponden a los países del Anexo I (o más desarrollados) (Müller et al, 2013). Las asimetrías per cápita identificadas son de al menos cinco órdenes de magnitud pues el promedio del consumo en los países del Anexo 1 es de 51 (-10/ +7 ) toneladas per cápita, mientras que en los países noAnexo 1 es de $10( \pm 1)$ toneladas per cápita (Ibid).

A nivel de asentamiento urbano, un primer cálculo de las dimensiones del stock de la Zona Metropolitana del Valle de México -ZMVM (Delgado, 2013) muestra la relevancia de tal ejercicio aproximativo en tanto que la contabilidad meramente de flujos directos es limitada, cuestión que puede tener implicaciones políticas importantes al sustentar medidas insuficientes. Y es que tan sólo la renovación anual del 1\% de la infraestructura de la ZMVM es equivalente, al menos, al 17\% de las emisiones de $\mathrm{CO}_{2} \mathrm{e}$ directas de dicho asentamiento en el año 2010 (Delgado, 2013). A ésas emisiones debe sumarse aquellas indirectas de la producción de alimentos, mismas que sólo para el caso de la carne, leche y huevos asciende entorno a un 29\% adicional para ese mismo año (Ibid). Así, las estimaciones de las actuales contribuciones de la ZMVM -y de otras- están clara y fuertemente subestimadas. ${ }^{9}$

Otros indicadores de (in)sustentabilidad, en cierto sentido de menor complejidad aunque aún así útiles y por tanto que vale la pena mencionar, son la huella ecológica o la huella hídrica en tanto permiten también dar cuenta de las implicaciones del acelerado proceso de urbanización experimentado en las últimas décadas del siglo XX y lo que va del siglo XXI. Así, por un lado, la huella ecológica permite advertir que para abastecerse y absorber sus emisiones, los asentamientos urbanos demandan una superficie territorial varias decenas de veces mayor a sus propias dimensiones, lo que implica que las ciudades se apropien de reservas ecológicas tanto nacionales como internacionales y que pueden o no ser compensadas a nivel de la $\mathrm{HE}$ global con las reservas ecológicas nacionales, excepto en el caso de aquellas ciudades de países con sobregiro ecológico (cuando la huella ecológica sobrepasa la biocapacidad de los ecosistemas nacionales).

8 - Ejemplificando, en 2008 el acero contribuyó con el 25\% de las emisiones industriales globales, el cemento 19\% y el aluminio el 3\%; en conjunto estos tres materiales representaron el $17 \%$ del emisiones relacionadas al uso total global de energía y de los procesos relacionados de emisión (en: Muller et al, 2013).

9 - Si bien hay otras emisiones indirectas que no son de algún modo contabilizadas en otros sectores (por ejemplo la electricidad usada en los sistemas de agua que sí es considerada dentro del sector energía como emisión directa), ésas se estiman, comparativamente hablando, de menor peso. 
De modo similar, la huella hídrica precisa volúmenes crecientes de consumo directo e indirecto de agua incluyendo aquella necesaria para asimilar la carga de contaminantes. El Cuadro 2 indica la huella ecológica del consumo y la biocapacidad disponible promedio en hectáreas globales (gha, por sus siglas en Inglés) de seis ciudades estudiadas. ${ }^{10}$ También precisa el prome-dio de sus huellas hídricas en sus tres dimensiones (azul, verde y gris). ${ }^{11}$

Es de subrayarse que en el caso de la Ciudad de México registraba en 1961 una huella ecológica de 8.7 millones de gha que, frente a su biocapacidad promedio disponible en ese año, se ubicaba en condición de superávit ecológico con 7.8 millones de gha. Una situación similar se registra con respecto a la (sobre) explotación de fuentes de agua, cuestión que se aborda con mayor detalle más adelante para el caso de la ZMVM en tanto caso único de la muestra con graves problemas y retos entorno a la disponibilidad, calidad y saneamiento del agua. El caso de Caracas, aunque la huella ecológica per cápita aumentó sólo alrededor de 0.7 gha per cápita de 1961 al 2007 , es notorio la dramática pérdida de biocapacidad, del orden de dos terceras partes, pues se pasó de 9.3 gha per cápita en 1961 a 2.89 gha per cápita en 2007.

Cuadro 2. Huella ecológica del consumo y huella hídrica del consumo de algunas ciudades latinoamericanas

\begin{tabular}{|c|c|c|c|c|c|c|c|}
\hline \multirow[t]{2}{*}{ Ciudad } & \multirow{2}{*}{$\begin{array}{l}\text { Huella } \\
\text { ecologica } \\
\text { (millones } \\
\text { de gha - } \\
2007 \text { ) }\end{array}$} & \multirow{2}{*}{$\begin{array}{l}\text { Sobregiro o } \\
\text { reserva } \\
\text { ecológica } \\
\text { (millones de } \\
\text { gha - 2007) }\end{array}$} & \multirow{2}{*}{$\begin{array}{l}\text { Veces de } \\
\text { superficie de } \\
\text { la ciudad } \\
\text { demandada en } \\
\text { Gha }-2007^{* *}\end{array}$} & \multicolumn{4}{|c|}{$\begin{array}{l}\text { Huella hídrica }(\mathrm{HH})-\text { millones de } \mathrm{m}^{3} / \text { año } \\
\text { (con base en el periodo } 1996-2005)^{* * *}\end{array}$} \\
\hline & & & & HH AzUI & HH verde & HH gris & HH Total \\
\hline $\begin{array}{l}\text { Ciudad } \\
\text { de } \\
\text { México }\end{array}$ & $\begin{array}{l}26.5 \\
\left(66^{*}\right)\end{array}$ & $\begin{array}{l}13.5 \\
\left(33.7^{*}\right)\end{array}$ & $\begin{array}{l}180 \\
\left(85^{\star}\right)\end{array}$ & $\begin{array}{l}1,683 \\
\left(4,184^{\star}\right)\end{array}$ & $\begin{array}{l}13,285 \\
\left(33,025^{\star}\right)\end{array}$ & $\begin{array}{l}2,537 \\
\left(6,307^{\star}\right)\end{array}$ & $\begin{array}{l}17,505 \\
\left(43,516^{\star}\right)\end{array}$ \\
\hline $\begin{array}{l}\text { Sao } \\
\text { Paulo }\end{array}$ & $\begin{array}{l}32.9 \\
\left(58.2^{\star}\right)\end{array}$ & $\begin{array}{l}68.6 \\
\left(121.4^{*}\right) \\
\end{array}$ & $\begin{array}{l}217 \\
\left(72^{\star}\right) \\
\end{array}$ & $\begin{array}{l}796 \\
\left(1,408^{\star}\right)\end{array}$ & $\begin{array}{l}20,407 \\
36,087 \\
\end{array}$ & $\begin{array}{l}1,722 \\
3,045 \\
\end{array}$ & $\begin{array}{l}22,925 \\
40,540 \\
\end{array}$ \\
\hline $\begin{array}{l}\text { Lima } \\
(+ \text { Callao }) \\
\end{array}$ & $\begin{array}{l}13.2 \\
\left(14.7^{\star}\right)\end{array}$ & $\begin{array}{l}19.9 \\
\left(22.2^{\star}\right)\end{array}$ & $\begin{array}{l}49.6 \\
\left(52.4^{\star}\right)\end{array}$ & $\begin{array}{l}1,305 \\
\left(1,454^{\star}\right)\end{array}$ & $\begin{array}{l}6,982 \\
\left(7,778^{\star}\right)\end{array}$ & $\begin{array}{l}1,088 \\
\left(1,212^{\star}\right)\end{array}$ & $\begin{array}{l}9,375 \\
\left(10,444^{\star}\right)\end{array}$ \\
\hline $\begin{array}{l}\text { Bogotá } \\
\text { D. C. }\end{array}$ & $\begin{array}{l}13.6 \\
\left(18.4^{\star}\right)\end{array}$ & $\begin{array}{l}15.4 \\
\left(20.8^{*}\right)\end{array}$ & $\begin{array}{l}85 \\
\left(106^{*}\right)\end{array}$ & $\begin{array}{l}487 \\
\left(656^{\star}\right)\end{array}$ & $\begin{array}{l}8,187 \\
\left(11,046^{*}\right)\end{array}$ & $\begin{array}{l}1,363 \\
\left(1,839^{*}\right)\end{array}$ & $\begin{array}{l}10,037 \\
\left(13,543^{\star}\right)\end{array}$ \\
\hline $\begin{array}{l}\text { Rio de } \\
\text { Janeiro }\end{array}$ & $\begin{array}{l}18.4 \\
\left(32^{\star}\right) \\
\end{array}$ & $\begin{array}{l}38.5 \\
\left(66.7^{\star}\right) \\
\end{array}$ & $\begin{array}{l}152 \\
\left(56^{\star}\right)\end{array}$ & $\begin{array}{l}447 \\
\left(774^{\star}\right) \\
\end{array}$ & $\begin{array}{l}11,457 \\
\left(19,848^{\star}\right)\end{array}$ & $\begin{array}{l}967 \\
\left(1,675^{\star}\right) \\
\end{array}$ & $\begin{array}{l}12,871 \\
\left(22,297^{\star}\right)\end{array}$ \\
\hline $\begin{array}{l}\text { Buenos } \\
\text { Aires }\end{array}$ & $\begin{array}{l}7.8 \\
\left(31.2^{\star}\right)\end{array}$ & $\begin{array}{l}14.7 \\
\left(58.8^{\star}\right)\end{array}$ & $\begin{array}{l}384 \\
\left(81^{*}\right)\end{array}$ & $\begin{array}{l}329 \\
\left(1,319^{\star}\right)\end{array}$ & $\begin{array}{l}3,971 \\
\left(15,881^{*}\right)\end{array}$ & $\begin{array}{l}521 \\
\left(2,083^{\star}\right)\end{array}$ & $\begin{array}{l}4,821 \\
\left(19,283^{\star}\right)\end{array}$ \\
\hline $\begin{array}{l}\text { Caracas } \\
\text { D.C. }\end{array}$ & $\begin{array}{l}6.1 \\
\left(8.8^{\star}\right)\end{array}$ & $\begin{array}{l}0.17 \\
\left(0.24^{*}\right)\end{array}$ & $\begin{array}{l}141 \\
\left(108.9^{\star}\right)\end{array}$ & $\begin{array}{l}186 \\
\left(268^{\star}\right) \\
\end{array}$ & $\begin{array}{l}2,980 \\
\left(4,282^{\star}\right)\end{array}$ & $\begin{array}{l}470 \\
\left(675^{\star}\right)\end{array}$ & $\begin{array}{l}3,636 \\
\left(5,225^{\star}\right)\end{array}$ \\
\hline Quito & $\begin{array}{l}3 \\
\left(4.1^{*}\right)\end{array}$ & $\begin{array}{l}0.7 \\
\left(0.9^{\star}\right)\end{array}$ & $\begin{array}{l}85 \\
\left(10^{\star}\right) \\
\end{array}$ & $\begin{array}{l}128 \\
\left(175^{\star}\right)\end{array}$ & $\begin{array}{l}1,691 \\
\left(2,325^{\star}\right)\end{array}$ & $\begin{array}{l}443 \\
\left(610^{\star}\right)\end{array}$ & $\begin{array}{l}2,262 \\
\left(3,110^{*}\right)\end{array}$ \\
\hline $\begin{array}{l}\text { Monte- } \\
\text { video } \\
\text { (+Canelo } \\
\text { nes y } \\
\text { San } \\
\text { José) }\end{array}$ & $\begin{array}{l}6.7 \\
\left(10^{\star}\right)\end{array}$ & $\begin{array}{l}6.3 \\
\left(9.5^{\star}\right)\end{array}$ & $\begin{array}{l}127 \\
\left(80.4^{*}\right)\end{array}$ & $\begin{array}{l}83 \\
\left(126^{\star}\right)\end{array}$ & $\begin{array}{l}2,580 \\
\left(3,912^{\star}\right)\end{array}$ & $\begin{array}{l}150 \\
\left(227^{\star}\right)\end{array}$ & $\begin{array}{l}2,813 \\
\left(4,265^{\star}\right)\end{array}$ \\
\hline \multicolumn{8}{|c|}{$\begin{array}{l}\text { * Estimación a escala metropolitana } \\
\star * \text { Número de veces de superficie territorial de la ciudad en cuestión que demanda su huella ecológica sin } \\
\text { descontar la biocapacidad, ello en tanto que ésa superficie también se subordina en otros espacios territoriales } \\
\text { a escala nacional. } \\
\star * \star \text { La huella hídrica se mide por periodos temporales para ajustar desviaciones de datos, sobre todo de huella } \\
\text { verde, derivados de periodos de secas o de lluvias abundantes. }\end{array}$} \\
\hline \multicolumn{8}{|c|}{$\begin{array}{l}\text { Nota: Se considera la biocapacidad per cápita disponible a escala nacional sin considerar la biocapacidad del } \\
\text { propio asentamiento urbano que tiende por lo general a ser negativa si se considera la pérdida de suelo de uso } \\
\text { de cultivo por el emplazamiento de infraestructura (que es como se considera metodológicamente en el } \\
\text { cálculo de la huella). Lo dicho se debe a que el suelo de conservación y zonas verdes siempre tienden a ser la } \\
\text { menor parte del territorio de las ciudades y porque su bioproductividad es siempre menor que la de suelos de } \\
\text { cultivo. En el caso de sobregiro ecológico nacional (caso de México), es claro que la HE restante se adquiere } \\
\text { vía el comercio internacional (en ese caso se trata de } 13.3 \text { millones de gha a escala de la ciudad y de } 31.5 \\
\text { millones de gha a nivel metropolitano). En el caso de tratarse de países acreedores o con reservas ecológicas a } \\
\text { escala nacional, se asume que tales reservas compensan la HE que en efecto se adquiere vía las importaciones. }\end{array}$} \\
\hline
\end{tabular}

10 - Las hectáreas globales es una unidad de medida común que permite estandarizar distintas superficies terrestres y marinas biológicamente productivas (de pesca, cultivos, pastizales, bosques, superficie construida, de captura de CO2e) con la media de bioproductividad mundial para un año dado.La bioproductividad, o producción primaria, es la cantidad y velocidad en la que ocurre la producción de biomasa en un ecosistema durante un periodo de tiempo determinado.

11 - La huella hídrica azul refiere al uso consuntivo del agua, incluyendo la que se evapora y que es incorporada al producto o servicio, así como la que no vuelve a la misma zona de flujo o en el mismo periodo temporal (dígase, época de lluvias y de secas). La huella hídrica verde es el agua de lluvia que usualmente se usa en la producción de alimentos. La huella hídrica gris es el volumen de agua dulce que se requiere para asimilar la carga de contaminantes con base en los estándares de calidad del agua ambiental existentes (léase: Mekonnen y Hoekstra, 2011). 


\section{La ecología política del metabolismo urbano}

El trabajo complejo e interdisciplinar propio del metabolismo urbano toma mayor sentido cuando ése se hibrida con el análisis de la dimensión económicopolítica y social, de estructuras de poder y asimetrías espaciales y temporales presentes (modelos extendidos del metabolismo de asentamientos humanos; Zhang, 2013), de ahí que Swyngedouw afirme adecuadamente que los flujos metabólicos circulatorios se gestan por medio de conductos sociales y biofísicos, contexto en el que los procesos de desterritorialización y reterritorialización (destrucción y reconstrucción del espacio construido) hoy por hoy sean definidos y estén en función de las posibilidades que tales o cuales espacios territoriales ofrezcan a la acumulación de capital (en Heynen,Kaika y Swyngedouw, 2005). En ese sentido, la función del territorio, se entiende como el espacio construido impregnado de la lógica o la función para la cual se estructura el territorio y en donde se gestan asimetrías propias de un desarrollo desigual (Harvey, 1996), contradicciones y procesos de resistencia.

Las resistencias sociales en pugna por un derecho a la ciudad, a una ciudad menos desigual, más sustentable, incluyente, equitativa y solidaria, dan cuerpo así a la denominada ecología política urbana en tanto que la pelea por tal derecho pasa por el derecho a la gestión del propio metabolismo urbano (Heynen, Kaika y Swyngedouw, 2005).

Así entonces y debido al arraigo de relaciones de poder dominantes, se observa la apropiación desigual de los flujos de energía y materiales, y del stock material, ello por medio de relaciones de mercado pero también de ciertas políticas públicas (dígase por ejemplo el gasto en transporte que tiende a privilegiar el de tipo privado muy por encima del público, ello por no hablar de movilidad; al respecto léase Delgado, coord., 2012). El resultado de una capacidad de compra desigual es que las mejores construcciones, los servicios de mayor calidad y el grueso del espacio público, cada vez más privatizado, es adjudicado a los "mejores" consumidores, es decir a las clases medias y altas, todo al tiempo que las externalidades negativas de la vida urbana tienden a exportarse en la medida de lo posible a los barrios periféricos o fuera de la ciudad (algo desde luego inviable para el caso de la contaminación atmosférica que afecta a la población de zonas geográficas por igual). ${ }^{12}$ En el proceso claramente las cuestiones de clase, género, etnicidad, entre otras, son centrales en términos de la capacidad de movilización de relaciones de poder para definir quién tiene acceso a, $o$ control de, y quién será excluido del acceso a, o el control de los recursos naturales y otros componentes del espacio urbano construido (Heynen, Kaika y Swyngedouw, 2005), incluyendo la imposición de los impactos socioambientales creados.
Desde tal perspectiva, por ejemplo, Delgado (2014) revisa el caso de la ecología política urbana en la ZMVM confirmando que la escases de recursos no sólo es biofísicamente definida sino socioeconómicamente construida, para lo cual es central ejercer y reproducir cuotas de poder (y dominación) asimétricas entre diversos actores, tanto públicos y privados para con el resto de la sociedad; se trata de formas de control que son sin embargo socialmente confrontadas de diversa manera y con distintas narrativas. Así, para el caso de la ZMVM, se identifican tres tipologías de conflictos entorno al acceso, gestión y usufructo del ciclo urbano del agua: (a) conflictos derivados por la apropiación de agua en cuencas aledañas a la ciudad; (b) conflictos interurbanos por el acceso a agua en cantidad y calidad, y (c) conflictos por afectaciones ambientales y a la salud por descargas de aguas residuales $y / o$ por el uso ésas para la producción de alimentos versus su tratamiento y reincorporación al ciclo urbano del agua (Delgado, 2014A). Otros ejercicios similares son los de Kaika y Swyngedouw (2002), Keil (2005) o los recopilados por Heynen, Kaika y Swyngedouw (2005), entre otros.

Lo relevante de hibridar los estudios de metabolismo urbano con la ecología política urbana es que, además de ayudar a calibrar mejor las medidas políticas y de regulación con respecto a las constricciones biofísicas y técnicas/tecnológicas reales o potenciales, también permite politizar el análisis de flujos y de stock material-energético, esto es, dando cuenta de la complejidad y diversidad de los procesos sociopolíticos incluyendo los contestatarios- que en una u otra forma modelan la ciudad. Es en tal sentido que Kaika y Swyngedouw (2002) sostienen que la ciudad puede ser vista como un "híbrido socionatural" compuesto por procesos metabólicos circulatorios heterogéneos.

Por tanto, la perspectiva hibrida en cuestión busca trascender los análisis convencionales al apostar por una visión que abrace la complejidad y dinamismo de los procesos biofísicos, económicos, sociopolíticos e histórico-culturales como un todo, ello en tanto que en la práctica modelan la ciudad.

Por todo lo antes descrito, se puede pues afirmar que la cuestión urbana vista desde una visión interdisciplinaria ya se desborda a y construye puentes entre diversos campos de conocimiento, muchos de ellos híbridos en sí mismos, tales como la mencionada ecología industrial, la economía ecológica, la ecología urbana y la ecología política. Desde cada interpretación se enfatizan rasgos diversos pero en conjunto se observa cómo ya se conforman lecturas cada vez más complejas en las que hay sinergias entre unos y otros acentos, dígase al tomar nota, tal y como dan cuenta Castán, Allen y Rapoport (2012: 851), de: (1) los flujos de materiales y de energía -así como el stock- de

12 - Los efectos no exportables a otros espacios, son sin embargo mejor afrontados o en su caso mitigados o neutralizados por aquellos individuos cuyo poder de compra es mucho mayor.

13 - Cabe precisar que otros autores también abordan la problemática de la ecología política urbana sin necesariamente hacer explícita la dimensión metabólica, no obstante los procesos que analizan por lo general suelen estar directamente vinculados a las dinámicas metabólicas y las asimetrías socioambientales presentes tanto hacia adentro de la ciudad como hacia fuera. 
las ciudades, (2) de la ciudad como ecosistema, (3) las relaciones económico-materiales dentro de las ciudades, (4) las relaciones campo-ciudad y sus impulsores económicos, (5) la reproducción de las inequidades urbanas, y (6) los intentos de darle otros significados a la ciudad a través de visiones novedosas en torno a las relaciones socioecológicas.

En ese sentido, lo que aquí se denomina como ecología política del metabolismo urbano se perfila claramente como campo híbrido en construcción que devela que la territorialización urbana y cualquier proceso de transición alternativo no es una cuestión neutral ni libre de las dinámicas termodinámicas y de las constricciones que de ésas se derivan.

\section{A modo de conclusión: el reto urbano y sus alternativas}

Reconociendo que efectivamente el conjunto de procesos económicos, políticos y socio-culturales a diferentes escalas espaciales y temporales, modelan $y$ remodelan el espacio-territorial urbano, sus perfiles metabólicos y sus implicaciones en relación directa a las relaciones imperantes de producción, puede sostenerse que la ecología política del metabolismo urbano, vista en positivo y subjetivamente materializada en la diversidad de movimientos sociales urbanos, puede ser punto de partida hacia imaginarios y prácticas pacíficas- que, con sentido de pertenencia, posibiliten configuraciones espaciales o tipologías territoriales alternativas, viables, más resilientes y justas. No obstante no hay un consenso claro en relación a si la ecología política -y consecuentemente la ecología política urbana- tiene entre sus objetivos la construcción de imaginarios puntuales y potenciales prácticas alternativas, aunque desde luego ofrece herramientas sobradas para el análisis del estado de situación, las asimetrías y estructuras de poder imperantes, la identificación de cuestiones o escenarios conflictivos y ciertamente de posibles rutas de solución (lo que no es estrictamente hablando lo mismo que construir imaginarios y potenciales prácticas, lo que entre otras cuestiones demanda abrazar la memoria histórica, incluyendo la geográfica-territorial, así como la diversidad sociocultural y sus implicaciones).

En todo caso, el necesario cambio de paradigma en la construcción, operación y gestión de las ciudades en cualquier parte del mundo deberá ser sin duda imaginativo y propositivo y sobre la base de una activa planeación integral entrópica tanto de las ciudades como de sus espacios rurales y sus áreas de conservación ya que pensar lo urbano de manera autocontenida no tiene sentido espacial-territorial alguno, ni socioeconómica, ni ambientalmente hablando. La suma de múltiples acciones, si ésas parten del mencionado proceso de planeación integral de los territorios y sus usos (incluyendo sus perfiles metabólicos), sugiere tener un mayor impacto al posibilitar sinergias y cobeneficios de diversa índole. Por ello, la tradicional gestión sectorializada ya no es suficiente, viable, ni deseable por mucho más tiempo.
El asunto circunscribe, además de la planeación integral de los usos del suelo, la valoración sobre la pertinencia y viabilidad de nuevas formas de diseño y construcción de infraestructura, el aprovechamiento de tecnologías genuinamente sustentables, la preservación de los cinturones verdes y la restauración ambiental de la periferia urbana, el cuidado y recuperación de ríos, canales y cuencas de inundación, el estímulo de programas formativos, informativos y de organización, participación y gestión ciudadana; entre otras acciones e instrumentos que permitan reconvertir las urbes, según la ruta que cada asentamiento se trace acorde a su propia realidad, en espacios cada vez menos devoradores de suelo, materiales y energía, más vivibles, aptos frente al cambio climático y la crisis medioambiental, y socialmente más justos e integradores (Álvarez y Delgado, 2014). Véase en la Figura 2 las principales tendencias en la literatura a cerca de las características clave propias de una asentamiento urbano "bajo" y alto en "carbono" a partir de integrar políticas públicas tanto de "sistemas" urbanos como de planeación espacial.

Para un éxito mayor, el análisis metabólico de los asentamientos urbanos se precisa de manera permanente de tal suerte que se puedan ajustar los instrumentos de política pública (y no meramente de gobierno) y las acciones concretas de múltiples agentes o actores a los cambiantes contextos imperantes y en relación a una tendencia histórica y un futuro socialmente deseable. No basta entonces con pensar el diseño del espacio urbano y de su perfil metabólico, es además nodal la reformulación de su función para que sea ésa el desarrollo del sujeto y no la acumulación de capital per se; la función es aquí entonces entendida en términos de relaciones sociales de producción y no en términos de zonificación, dígase de usos del suelo (comercial, industrial, residencial, de conservación).

Estamos ante una apuesta que no pasa por meras soluciones tecnológicas o de tipo y diseño de infraestructura, sino de un cambio a fondo de la lógica imperante y por tanto de la naturaleza y deseabilidad de las propias soluciones. Es un contexto en el que, no sobra decirlo, ninguna receta es generalizable aún cuando se reconozcan como deseables ciertas nociones generales (los formatos e imaginarios de ciudad y eventuales soluciones no deben, ni pueden ser siempre estandarizados). Diversas propuestas que hoy día se vienen configurando para la conformación de ciudades "resilientes", "sustentables", "bajas en carbono", "verdes", etcétera, bien pueden ser en alguna medida' útiles, pero en sí mismas no son suficientes si no parten de nuevos paradigmas a cerca tanto de la forma como de la función de los espacios construidos. Ello implica no sólo un profundo rompimiento epistemológico con las ideas dominantes, sino en el fondo una apuesta por otras modernidades, emancipadoras, con equidad y libres del yugo del mercado, esto es, un cambio concreto del sistema de producción y reproducción de la humanidad. 
Figura 2. Características clave de asentamientos urbanos de bajo y alto carbono.

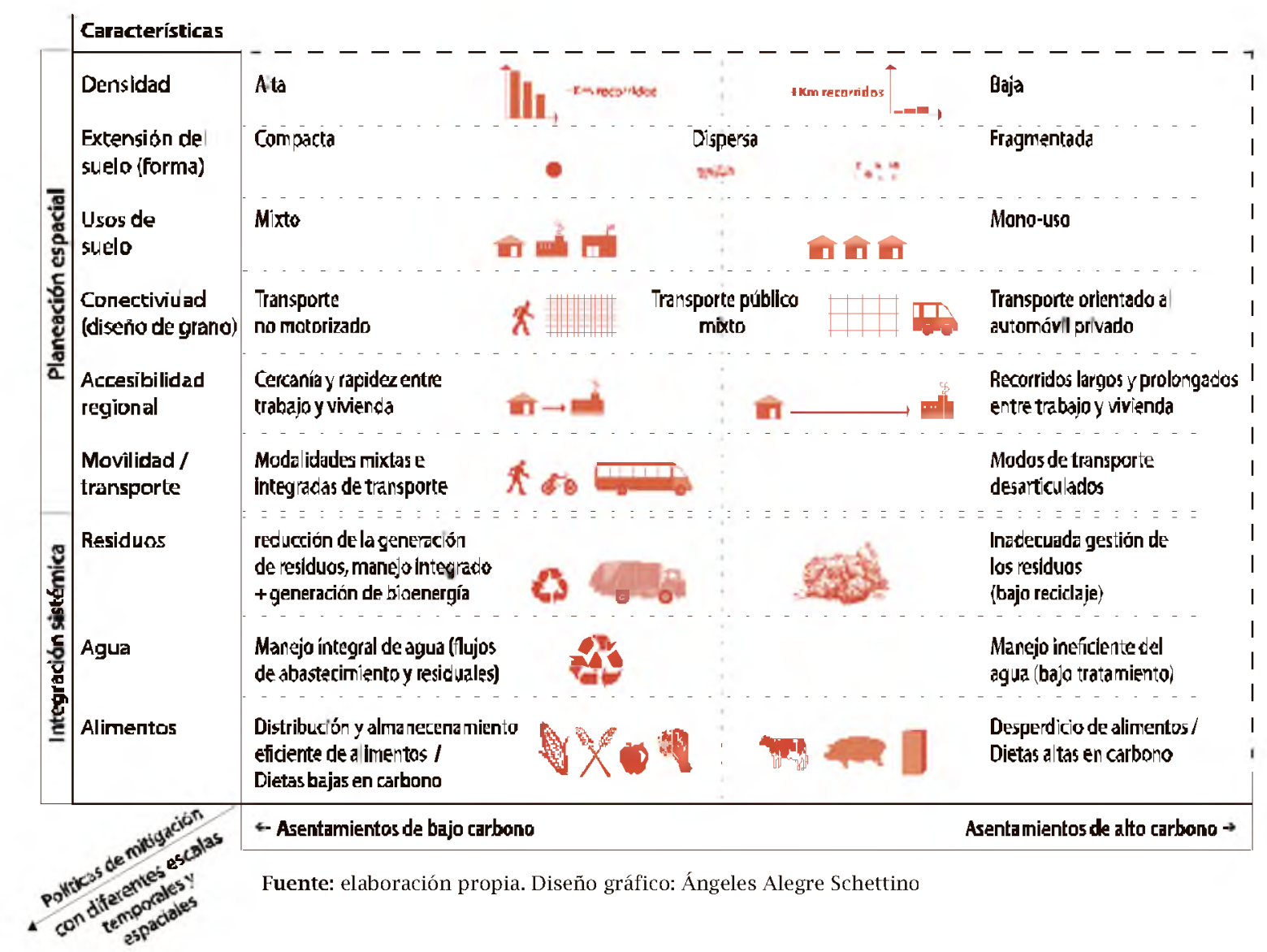

La configuración del espacio desde una perspectiva nacional alternativa que articule lo urbano y lo rural, requiere no sólo de la (re)distribución más equitativa de la riqueza, sino de la reconstitución de la base productiva -en especial la local y nacional-, ahora avocada a la producción de valores de uso para la buena vida y para el consumo interno (cada vez más de tipo local y regional) y por tanto alejada de la producción de valores intrínsecamente nocivos y de cualquier planteo de economías de exportación, típicamente extractivas y de enclave.

Se trata de un diseño que además prioriza la soberanía energética y alimentaria y la cobertura total de servicios básicos, incluyendo la salud (fortaleciendo tanto lo preventivo como lo curativo); que desarrolla responsablemente la ciencia y las tecnologías que no contradicen el bien común de la humanidad y el derecho de existencia de otras especies (el valor intrínseco de la naturaleza); que recupera la ciencia no-validada bajo los criterios de evaluación imperantes en el actual sistema académico de evaluación por pares, así como los conocimientos tradicionales que resulten útiles en la construcción de imaginarios y soluciones alternativas; que busca modalidades productivas y establece criterios para el uso racional de los recursos; que exige condiciones ambientales óptimas y por consiguiente que respeta estrictamente las fronteras ecológicas; que aboga por la disminución del metabolismo social en especial de parte de los espacios urbanos de países ricos, dígase a partir de hacer prohibitivo todo derroche de energía y materiales, aumentar el tiempo de vida de los productos, e incrementar el reciclaje y re-uso de los materiales, entre otras medidas.

Es un panorama en el que la transformación de los territorios a escala de lo local requiere del resurgimiento de los valores territoriales a esa escala, esto es a decir de Magnaghi (en: Palacio, 2012), las identidades del lugar, en especial aquellas de larga duración y las prácticas cotidianas, todo con el objeto de favorecer, precisa, la reterritorialización del "desarrollo", este último pensado en términos genuinamente alternativos. Lo dicho en efecto obliga imaginar colectivamente procesos y acciones de transición y de cambio de paradigma dando cuenta de la viabilidad en el cortomediano plazo, y para cada caso y contexto específico, pero incluso también a replantear las relaciones de poder -o de gestión- más adecuadas para ello, un asunto que precisaría toda una nueva institucionalidad y normatividad para el bien común. En este tenor, la construcción de alternativas genuinas y caminos de transición no puede ser más que producto de un ejercicio de coproducción de conocimiento, complejo 
e interdisciplinar, de ciencia reflexiva (Ungar y Strand, 2005) y, sobre todo, altamente participativa (Funtowicz y Strand, 2007A; Funtowicz y Strand, 2007B).

\section{Referencias}

Álvarez Enríquez, Lucía y Delgado Ramos, Gian Carlo, 2014. "Ciudades, gestión territorio y ambiente". Interdisciplina. Revista del Centro de Investigaciones Interdisciplinarias en Ciencias y Humanidades. Vol. 2. No. 2. UNAM. México. Enero - Abril.

Angel S., J. Parent, D.L. Civco, A. Blei, and D. Potere. 2011. "Thedimensions of global urbanexpansion: Estimates and projectionsforallcountries, 2000-2050." Progress in Planning. 75. Pp. 53-107.

Ayres, Robert. 1994. "Industrial metabolism: theory and policy", en: Ayres, R. y Simonis, U.K. (eds.). Industrial Metabolism: RestructuringforSustainableDevelopment.UnitedNationsUniversi tyPress. Tokyo, Japón. pp. 3-20.

Baccini, P., y Brunner, P. 1990. Metabolism of theAnthroposphere. FirstEdition. MIT Press. Cambridge, MA. / Londres.

Baccini, P., y Brunner, P. 2012. Metabolism of theAnthroposphere SecondEdition. MIT Press. Cambridge, MA. / Londres.

Barles, Sabine. 2009. "Urban Metabolism of Paris and Its Region." Journal of Industrial Ecology. Vol. 13. No. 6. Pp. 898-913.

Barles, Sabine. 2007. "Feeding the city: food consumption and flow of nitrogen. Paris 1801-1914. "Science of the Total Environment. Vol. 375. No. 1-3.Pp. 48-58.

Brunner, Paul y Rechberger, Helmut. 2005. PracticalHandbook of Material FlowAnalysis. Lewis Publishers. Florida, EUA.

Castán Broto, Vanesa., Allen, Adriana y Rapoport, Elizabeth. 2012. "Interdisciplinaryperspectivesonurbanmetabolism". Journal of Industrial Ecology. Vol. 16. No. 6. Pp. 851 - 861.

Daiber, Birgit y Houtart, Francois (eds). 2012.Un paradigma poscapitalista: el bien común de la humanidad.Panamá/Cuba, Ruth Casa Editorial.

Delgado-Ramos, Gian Carlo. "Ciudad, agua y cambo climático: una aproximación desde el metabolismo urbano." Medio Ambiente y Urbanización. No. 80. Buenos Aires, Argentina. IIED-América Latina.

Delgado-Ramos, Gian Carlo. 2013. "Climatechange and metabolicdynamics in Latin American majorcities" en:Zubir, S.S. y Brebbia, C.A. (editores). Sustainable City VIII. UrbanRegeneration and Sustainability. WIT Press. Southampton, Reino Unido., Pp. 39 56.

Delgado-Ramos, Gian Carlo., Campos Chávez, Cristina., y Rentería Juárez, Patricia. 2012. "Cambio climático y el metabolismo urbano de las megaurbes latinoamericanas". Hábitat Sustentable. Vol. 2. No. 1. Santiago de Chile, Chile. Pp. 2 - 25.

Delgado-Ramos, Gian Carlo. 2011. Imperialismo Tecnológico y Desarrollo en América Latina. La Habana / Panamá, Ruth Casa Editorial.

Delgado Ramos, Gian Carlo. Coord. 2012. Transporte, ciudad y cambio climático. CEIICH-UNAM. México.

Delgado-Ramos, Gian Carlo. Coord.. 2014. Buena Vida, Buen Vivir: imaginarios alternativos para el bien común de la humanidad. CEIICH, UNAM. México.

Davis, M. 2006. Planet of Slums.London-Nueva York. Versus.

Díaz Álvarez, C.J. 2011. Metabolismo de la Ciudad de Bogotá: una herramienta para el análisis de la sostenibilidad ambiental urbana. Universidad Nacional de Colombia. Bogotá, Colombia.

Ewing, B., Moore, D., Goldfinger, S., Oursler, A., Reed, A., y Wackernagel, M. 2010. TheEcologicalFootprint Atlas 2010. Global Footprint Network. Oakland, California., EUA.

Færge, Jenas, Magid, Jakob, y Penning de Vries, Frits.W.T. 2001. "Urban nutrient balance for Bangkok." Ecological Modelling. Vol. 139. No.1.Pp. 63-74.

Forkes, Jennifer. 2007. "Nitrogen balance for the urban food metabolism of Toronto, Canada." Resources, Conservation \& Recycling.Vol. 52.No. 1.Pp. 74-94.

Funtowicz, Silvio y Strand, Roger. 2007A. "De la demostración experta al diálogo participativo". Revista CTS. No. 8. Vol. 4. Pp. 97-113.

Funtowicz, Silvio y Strand, Roger. 2007B. "Models of Science and Policy" en: Traavik, T. y Lim, L.C. (eds). BiosafetyFirst - Holistic Approachesto Risk and Uncertainty in Genetic Engineering and Genetically Modified Organisms. Tapir Academic Press, Trondheim, Noruega.

Gandy, Matthew. 2004. "Rethinking urban metabolism: water, space and the modern city." City. Vol. 8. No. 3. Pp. 364-379.
Haberl, Helmut. 1997. "Human appropriation of net primary production as anenviron mental indicator: implications for sustainable development." Ambio. Vol. 26. No. 3. Pp. 143 - 146.

Haberl, Helmut., 2001. "The energetic metabolism of societies: part I: accounting coccepts." Journal of Industrial Ecology. Vol. 5. No. 1. Pp. 11-33.

Hanya, T., y Ambe, Y. 1976. "A study on the metabolism of cities", en: Science for a better environment: proceedings of the International Congress on the Human Environment. Tokyo, Japón. Pp. 228- 233

Harvey, David. 1996. Justice, nature and geography of difference. Oxford, Reino Unido. Blackwell.

Hermanowicz, Slawomir W, Asano, Takashi. 1999. "Abel Wolman's 'The Metabolism of Cities' re-visited: a case for water recycling and reuse." Water Science \& Technology. Vol. 40. No. 4-5. Pp. 29-36.

Heynen, N., Kaika, M., y Swyngedouw, E., M. 2005. "Urban Political Ecology - Politicising the Production of Urban Natures", en: In the Nature of Cities - Urban Political Ecology and the Politics of Urban Metabolism. Routledge. Londres, Reino Unido.

IPCC - Intergovernmental Panel on Climate Change. Summary for Policymakers. Working Group III contribution to the IPCC Fifth Assessment Report (AR5). Climate Change 2014: Mitigation of Climate Change. Sesión 39 del IPCC. 7-12 de Abril, 2014. Berlín, Alemania.

INECC. 2012. Diagnóstico Básico para la Gestión Integral de los Residuos. INECC-SEMARNAT. México.

Kaika, Maria y Swyngedouw, Erik. 2002. "Fetishizing the modern city: thephantasmagoria of urban technological networks". Urban and Regional Research. Vol. 24. No. 1.Pp. 120-138.

Kennedy, C., Cuddihy, J., y Engel-Yan, J. 2007. The Changing Metabolism of Cities. Journal of Industrial Ecology, 11(2), 43-59.

Kennedy, C. Steinberg, J., Gasson, B., Hansen, Yvone., Hillman, Timothy., Havránek, M., Pataki, D., Phdungsilp, A., Ramaswami, Anu y Villalba Méndez, G. 2009. "methodology for inventoryinggreen house gas emissions from global cities. Fnergy Policy. Vol. 38. Elsevier. Pp. 4828-4837.

Intendencia de Montevideo. 2009. Informe Ambiental de Montevideo 2009. Montevideo, Uruguay.

Intendencia de Montevideo. 2013. Inventario de Emisiones de Gases de Efecto Invernadero 2006 - 2008 - 2010. Montevideo. Montevideo, Uruguay.

Jiménez, Mayerling. 2012. "Alcaldía de Caracas recolectó 939 mil toneladas de desechos sólidos en 2011." Radio Mundial. Venezuela. En línea [19/08/14]:

www.radiomundial.com.ve/article/alcald\%C3\%AD-de-caracasrecolectó-939-mil-toneladas-de-desechos-sólidos-en-2011

Kennedy, C., Pinceti, S., y Bunje, P. 2011. "Thestudy of urban metabolism and itsapplicationsto urban planning and design." EnvironmentalPollution. Vol. 159, 8-9. Pp.1965 - 1973.

Leach, M.A., Bauen, A., y Lucas, NID. 1997. "A Systems Approach to Materials Flow in Sustainable Cities: a case study of paper." Journalof Environmental Planning and Management. Vol. 40. No. 6. Pp. 705-723.

Lefebvre, H.. 1976. Thesurvaival of Capitalism: reproduction of therelations of production. Allison \&Busby. Londres, Reino Unido.

Lehmann, Steffen. 2011. "Optimizing urban material flows and waste streams in urban development through Principles of Zero Waste and Sustainable Consumption." Sustainability. Vol. 3. No. 1. Pp. 155-183.

Mekonnen, M.M. y Hoekstra, A.Y. 2011. National water foot print accounts: The green, blue and grey waterfoot print of production and consumption. Value of Water Research Report Series No. 50, UNESCO-IHE, Delft, Holanda.

Ministerio del Medio Ambiente y Recursos Naturales. 2005. Primera Comunicación Nacional en Cambio Climático de Venezuela. República Bolivariana de Venezuela / GEF. En línea [20/8/14]: http:/ unfccc.int/resource/docs/natc/vennc01.pdf

Minx, Jan., Creutzig, Felix., Medinger, Verena., Ziegler, Tina., Owen, Anne., y Baiocchi, Giovanni. 2010. Developing a pragmaticapproachtoassessurbanmetabolis in Europe. A report to the European Environment Agency. Stockholm Environment Institute / Universidad Técnica de Berlín.

Müller, Daniel B., et al. 2013. "Carbon Emissions of Infrastructure Development." Environmental Science \& Technology. Vol. 47. Pp. $11739-11746$.

Mumford, L. 1961. The City in History: ItsOrigins, ItsTransformations and ItsProspects. Nueva York, Estados Unidos. Harcourt, Brace\&World.

Naciones Unidas. 2011. World Urbanization Prospects, the 2011 Revision. The Population Division of the Department of Economic and Social Affairs of the United Nations.

Newcombe, Ken, Kalma, Jetse D., y Aston, Alan R. 1978. "The 
metabolism of a city: the case of Hong Kong." AMBIO Journal of Human the Environment Vol. 7. No. 3. Pp. 3-15.

Palacio Tamayo, Dolly Cristina. 2012. "Reseña de 'El proyecto local' de Alberto Magnaghi." Territorios. No. 26. Universidad del Rosario. Colombia. pp. 135-143.

SEDAPAL. 2013. Plan Estratégico de Tecnologías de la Información y Comunicaciones $2009-2013$. En línea [20/08/14]:www.sedapal.com.pe/c/document_library/get_file?u uid $=f c 3823 d 4-59 b 2-4 b 7 d-a e c 0-35 c a 798 b 2 e 9 e$

Seifert, Reinhard. 2009. Análisis de la situación del agua (cantidad y residual) en Lima Metropolitana. Proyecto LiWa/Zirn-SEDAPAL. Lima, Perú. En línea [20/08/14]: www.limawater.de/documents/rseifert_studie.pdf

SMA-DF - Secretaría de Medio Ambiente del Distrito Federal. 2012. Inventario de Emisiones de la Zona Metropolitana del Valle de México, 2010. Gobierno del Distrito Federal. México.

Thériault, Julie, Laroche, Anne-Marie. 2009. "Evaluation of the urban hydrologic metabolism of the Greater Moncton region, New Brunswick." Canadian Water Resources Journal. Vol. 34. No. 3. Pp. 255-268.

Toledo, Victor M., Alarcón-Cháires, Pablo., Barón, Lourdes. "Revisualizar lo rural desde una perspectiva multidisciplinaria". Polis. Revista de la Universidad Bolivariana. Vol. 8. No. 22. Pp. 329 345. En línea: www.scielo.cl/pdf/polis/v8n22/art19.pdf

UN-HABITAT. 2009. PlanningSustainableCities. Global Reporton Human Settlements 2009. Earthscan. Londres, Reino Unido.

Ungar, Paula y Strand, Roger. 2005. "Complejidad: una reflexión dese la ciencia de la conservación". Nómadas. No. 22. Universidad Central de Colombia. Colombia. Pp. 36-46.

Wolman, A. 1965. "Themetabolism of cities." Scientific American 213(3): 179-190.

Zhang, Y. 2013 . "Urbanmetabolism: a review of researchmethodologies." EnvironmentalPollution. No. 178. Pp. $463-473$

Zucchetto, J. 1975. "Energy, economictheory and mathematicalmodelsforcombiningthesystems of man and nature. Case study, theurbanregion of Miami." EcologicalModelling, 1, pp. 241-268. 